\title{
Saliva substitute mouthwash in nasopharyngeal cancer survivors with xerostomia: a randomized controlled trial
}

\author{
Dayaabaran Marimuthu ${ }^{1} \cdot$ Kong Min Han ${ }^{1} \cdot$ Mohd Shawal Firdaus Mohamad ${ }^{2} \cdot$ Mawaddah Azman $^{1}$ (i)
}

Received: 13 August 2020 / Accepted: 7 October 2020 / Published online: 11 November 2020

(C) The Author(s) 2020

\begin{abstract}
Objectives Xerostomia is a prevalent sequelae among nasopharyngeal cancer (NPC) survivors; yet, effective treatment protocols have been elusive. This study was a prospective randomized clinical trial to compare the effects of saliva substitute mouthwash in nasopharyngeal cancer survivors with xerostomia, between two treatment arms, conducted in a tertiary center.

Materials and methods This study measured the effects within 4 weeks in relation to summated xerostomia inventory (SXI) and unstimulated whole saliva (UWS). Patients randomized into the interventional arm were prescribed an immunologically active saliva substitute (IASS), while patients in the control arm were prescribed a non-immunologically active mouthwash as placebo. Results The study population consisted of 94 patients. There was a significant difference in SXI difference $(p<0.0001)$ and UWS difference $(p<0.0001)$ between control and interventional arms. No harmful side effects associated with the use of either mouthwash encountered throughout the study duration.

Conclusion IASS mouthwash significantly reduces subjective xerostomia scores measured using SXI and improves objective measurement of salivary flow using UWS among nasopharyngeal cancer survivors with xerostomia.

Clinical relevance IASS is significantly more effective in improving subjective and objective xerostomia measurements compared to non-immunologically active mouthwash. Additionally, this treatment is very safe, with superior side effect profiles.

Trial registration ClinicalTrials.gov Identifier: NCT04491435
\end{abstract}

Keywords Xerostomia $\cdot$ Mouth dryness $\cdot$ Hyposalivation $\cdot$ Saliva artificial $\cdot$ Saliva substitute $\cdot$ Mouthwashes $\cdot$ Radiation-induced toxicity $\cdot$ Nasopharyngeal cancer

\section{Introduction}

Nasopharyngeal carcinoma (NPC) is the highest reported otorhinolaryngological malignancy in Malaysia affecting predominantly male adults between 40 and 60 years old $[1,2]$. Radiation therapy (RT) has been coined as the mainstay treatment owing to its' radiosensitive properties [1,3]. Radiationinduced DNA damage impairs proper cell division, resulting

Mawaddah Azman

mawaddah1504@yahoo.com

1 Department of Otorhinolaryngology and Head and Neck Surgery, Level 9, Clinical Block, UKM Medical Centre, National University of Malaysia Kuala Lumpur Campus, Jalan Yaacob Latiff, Bandar Tun Razak, 56000 Kuala Lumpur, Malaysia

2 Centre of Studies for Oral and Maxillofacial Surgery, Faculty of Dentistry, UiTM Sungai Buloh Campus, Jalan Hospital, 47000 Sugai Buloh, Selangor, Malaysia in cell death or senescence of cells that attempt to divide, particularly useful in killing malignant cells. However, radiation doses to the salivary glands cause loss of saliva producing acinar cells which ultimately hampers production of saliva in NPC patients post radiation [4]. This leads to progressive loss of salivary gland function causing xerostomia symptoms [5].

Xerostomia, or dry mouth caused by reduced or absent saliva flow, is a subjective symptom that can lead to impaired chewing, swallowing, altered sense of taste, and speech. This eventually affects their nutritional status and quality of life. The reported prevalence of xerostomia in NPC survivors ranged from 80 to $100 \%$ [6-10]. This high prevalence has stemmed a lot of interest in prevention and treatment of this important sequelae. Palliative treatments such as mucosal lubricants, saliva substitute, saliva stimulants, and systemic or local sialogogues have been proposed [11-13].

Saliva substitute can be classified based on its active ingredients: carboxymethylcellulose, glycerol, immunologically active saliva substitute, xanthan, and herbal preparations 
[14]. They are commercially available in the forms of gels, toothpaste, sprays, or mouthwash [15]. Given that a wide variety of saliva substitute preparations are available, either as prescriptions or over the counter medications, good quality randomized trials are necessary to investigate its effectiveness in specific patient populations. A recent review in 2019 on saliva substitute included 21 studies conducted on various patient populations, a mere 4 trials in radiation-induced xerostomia and only 2 non-randomized trials on immunologically active saliva substitute [14]. The paucity of data on clinical effectiveness of saliva substitute in patients with radiation-induced xerostomia is rather compelling and warrants further research.

Oral7 ${ }^{\circledR}$ mouthwash (Oral7 International, UK) is an immunologically active saliva substitute (IASS) formulated with natural enzymes such as lactoperoxidase, lysozyme, glucose oxidase, and lactoferrin, similar to naturally occuring saliva. The biophysical properties of the mouthwash can potentially provide relief to xerostomia symptoms in patients post radiotherapy translating to a better quality of life. Hence, the purpose of the present study is to evaluate the effects of IASS in treating xerostomia among NPC patients post radiotherapy. The primary end-point of the study was to compare the subjective xerostomia symptoms, measured using a validated inventory taken 4 weeks following intervention and at baseline between patients who did not receive and patients who received Oral7® mouthwash. The secondary end-point was to compare unstimulated whole salivary flow measurements using sialometry technique between patients who did not receive and patients who received Oral7® mouthwash after a treatment duration of 4 weeks.

\section{Materials and methods}

The efficacy of the saliva substitute Oral7® mouthwash was evaluated in a prospective, double-blind, randomized, placebo-controlled study that was conducted in the Department of Otorhinolaryngology, Universiti Kebangsaan Malaysia Medical Centre, a tertiary referral center between June 1, 2018 (date of first patient recuitment) and January 2, 2020 (date of last patient follow-up) for a duration of 19 months. The study received partial funding from the Fundamental Grant of National University of Malaysia and the local distributor provided the Oral7® mouthwash used in this study. This study was approved by the Institutional Review Board of National University of Malaysia, with the project code FF-2018-133, along with its financial support on May 5, 2018. The conduct of the study followed the Declaration of Helsinki 1964 and the authors have no financial or affiliations with the distributors of the product to disclose. This study was designed, analyzed, and interpreted according to the CONSORT protocol (Fig. 1) [16].
All consecutive patients diagnosed with nasopharyngeal carcinoma during the study period who underwent either radiotherapy or concurrent chemo-radiotherapy were screened for the study. Patients who satisfied the inclusion and exclusion criterias (Table 1) were recruited. Written informed consent was obtained from the patients for publication of this manuscript. A copy of all the written consents is available for review by the Editors-in-Chief of this journal.

A randomization list was generated from the statistics website http://www.graphpad.com/quickcalcs/index.cfm where consecutive patients enrolled into the study were randomized to intervention and control arms with an allocation ratio of $1: 1$. The randomization list was generated by the lead researcher, who was not involved in enrolling, assigning the patients, and collection of data. The researcher assigning the participants to intervention arms did not know the allocation sequence until the moment of assignment. This way, the researcher was prevented from (consciously or unconsciously) influencing which participants who were assigned to a given intervention arm. Patients were dispensed sample A (saliva substitute containing Oral7 ${ }^{\circledR}$ mouthwash) or sample B (placebo containing Colgate Plax ${ }^{\circledR}$ ), in similar non-pressurized pumps (Fig. 2) according to the randomization list.

Oral7 ${ }^{\circledR}$ mouthwash (Oral7 International, UK) is an immunologically active saliva substitute (IASS) formulated with natural enzymes such as lactoperoxidase, lysozyme, glucose oxidase, and lactoferrin, similar to naturally occuring saliva. The mouthwash in liquid form was repackaged into specific non-pressurized pumps measuring $90 \mathrm{~g}$ each and was labeled sample A. Sample A was dispensed to patients recruited to the interventional arm.

Colgate Plax® (Colgate-Palmolive, Malaysia) is a mouthwash with biocidal properties containing cetylpyridinium chloride as the main active ingredient. This mouthwash was with no alcohol, no mucin, and no natural enzymes, making it a suitable placebo for this study. Additionally, the mouthwash contains xylitol, which provides some moisturizing effects, suitable for use in NPC survivors with xerostomia. The mouthwash in liquid form was repackaged into specific nonpressurized pumps measuring $90 \mathrm{~g}$ each and was labeled sample B. Sample B was dispensed to patients recruited to the control arm. Samples A and B possessed similar physical characteristics, such that the patients were blinded to the intervention that they received (Fig. 2).

The samples were dispensed by a dedicated nurse who was not part of the study. Both the researcher and patient are blinded to the type of intervention they received. The randomization list was not known to the researcher measuring the study outcome measures until data was analyzed. Patients were instructed to use the mouthwash by spraying 2 puffs of either samples A or B, three times a day. They were instructed to discard the samples if it had discolored or contained 


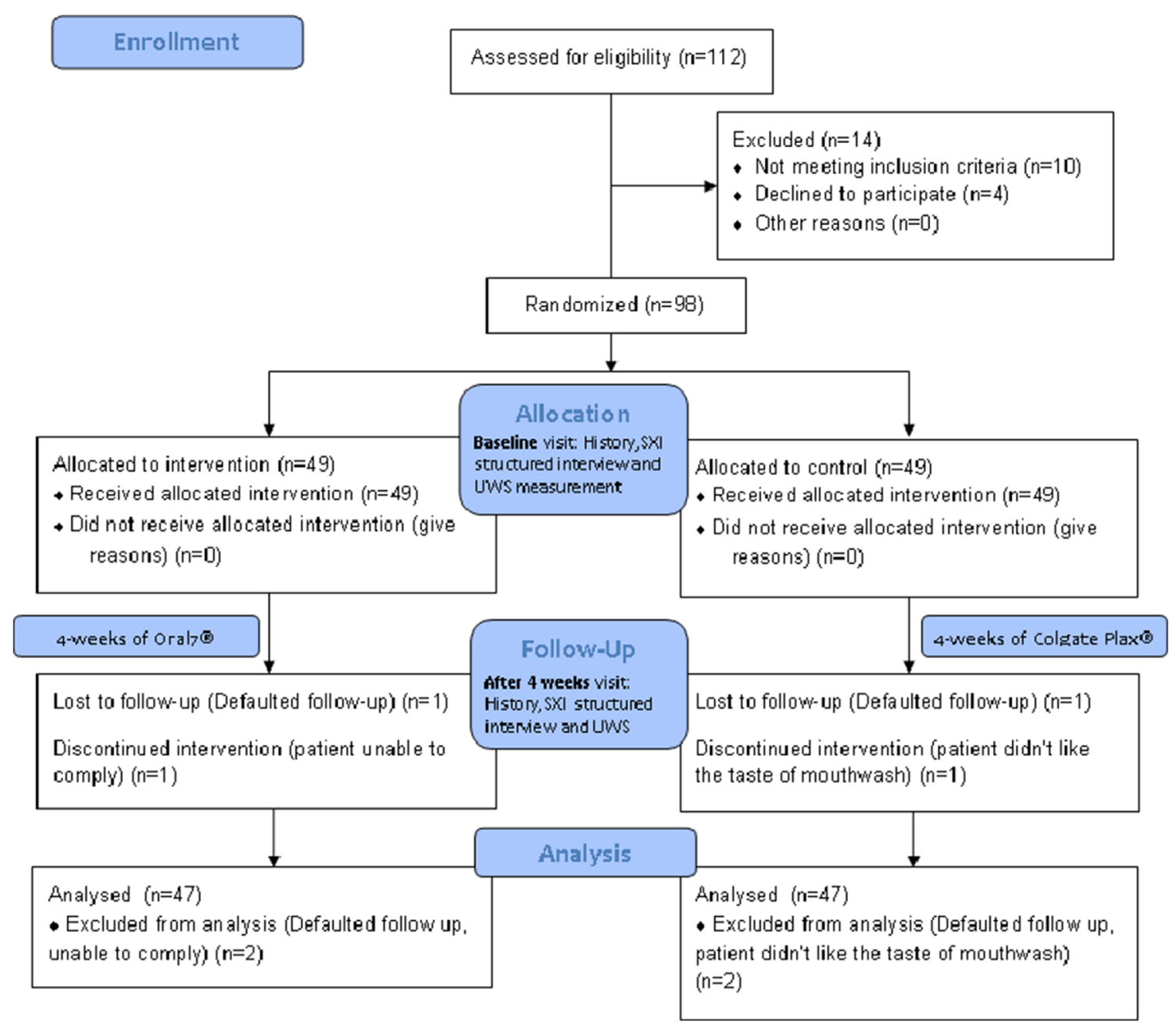

Fig. 1 Study flow

particulate material. Side effects experienced were collected through patient interviews either during weekly follow-up phone calls or clinic visits. The patients were not allowed to use other mouthwashes and hormonal or nutritional supplements throughout the study period. At the first visit, patients were given 2 weeks' supply of mouthwash in a designated box. Patients were instructed to bring the designated box with the remaining samples at the subsequent 2-week visit. They were told that more than enough samples were dispensed in order to encourage them to return unused samples. The number of samples left in the box and the weight of samples in each non-pressurized pump were measured at each visit and freshly measured samples were replenished at the end of 2 weeks. To further ensure patient compliance, weekly phone calls were made to all patients.

Demographic data including age, gender, education level, occupation, date of treatment completion, and relevant medical history such as type of treatment, Karnofsky performance score, histopathological type, and underlying medical illness were recorded. At the first visit, all patients underwent a structured interview by a dedicated researcher to obtain a baseline score of the summated xerostomia inventory (SXI). During the same visit, unstimulated whole saliva (UWS) flow was assessed by the same dedicated researcher using sialometry technique. All patients were instructed to use the saliva substitute (sample A) or placebo (sample B) for 4 weeks and the SXI and UWS were reassessed 4 weeks later. The flow of the study is summarized in Fig. 1. Follow-up phone calls were performed on a weekly basis to ensure compliance and record potential side effects that the patients may have experienced when using either samples A or B. Weight of returned empty pumps were documented to record compliance in recruited patients.

\section{Sample size calculation}

The sample size calculation for this interventional study was performed using Power and Sample Size (PS3) software (2009) by Dupont and Plummer from Vanderbilt University. The method used was the formula for prospective studies with dichotomous outcome and analyzed by $t$ tests. Ninety-four 
Table 1 Inclusion and exclusion criteria

\section{Inclusion criteria}

1. Patients who have completed radiation therapy or concurrent chemotherapy and radiation therapy for nasopharyngeal carcinoma (clinical staging of tumor based on AJCC staging ( $8^{\text {th }}$ edition) $T_{1-4}$, $\left.N_{0-3}, M_{0}\right)$.

2. Patients age 20 years old to 85 years old.

3. Two months has elapsed since last dose of chemotherapy or radiotherapy.

4. Karnofsky performance score more than $70 \%$.

5. Patients complaining of xerostomia.

Exclusion criteria

1. Those contraindicated to using mouthwash (established allergy to lactoperoxidase, lysozyme, glucose oxidase lactoferrin, cetylpyridinium chloride, and xylitol).

2. Patients with residual or recurrent disease.

3. Patients who received intensity-modulated radiation therapy.

4. Patients with ongoing oral mucositis (WHO Oral Mucositis grading I to IV).

5. Patients with facial, glossopharyngeal. vagus and hypoglossal nerve palsy/ paresis.

6. Patients who had any form of concurrent treatment protocols (hormonal, alternative, antiviral, sialogogues, or photodynamic therapy) during the study duration.

7. Patients with autoimmune diseases such as systemic lupus erythematosus, rheumatoid arthritis, and Sjớgren syndrome.

patients with 47 patients from the interventional population were needed to make the study statistically significant with a $95 \%$ confidence interval to detect significant differences between the control and interventional groups.

\section{Data collection and analysis}

Demographic data, including patients' age, sex, and race, were obtained from patient interviews. Cancer type, staging, and oncologic treatment performed were obtained from patients' records, radiological reports, or the final pathological report from the Health Information System. Patients' age was grouped according to Erik-Eriksson's psychological stages of life [17]. Tumors were staged based on the American Joint Committee for Cancer (8th Edition) [18].

Subjective xerostomia score was measured using summated xerostomia inventory (SXI). The Dutch version of the SXI was used as a primary outcome measure. The SXI contained 5 questions and was chosen as a more suitable questionnaire compared to the longer xerostomia inventory (XI) with 11 questions. Thomson et al. looked at different studies that compared the validity of SXI to XI which showed properties of the scale were not compromised by reducing the number of response options available to respondents. The authors suggested that the shorter measure has considerably better face validity than the original, because the items were more salient [19]. The SXI score was assessed in a structured interview by a dedicated researcher, each question provided in Table 2. For each question, patients were instructed to choose one of three responses to describe their subjective response past one week, either "Never" (score 1), "Occasionally" (score 2), and "Often" (score 5). The total score was calculated for both baseline and 4 weeks following intervention visits. Total scores ranged from 5 (no xerostomia) to 25 (worst possible xerostomia). The difference between the total SXI score taken at baseline and after 4 weeks of intervention was calculated and used as an outcome measure.

Sialometry was performed by a trained dedicated researcher, at the beginning of the study and 4 weeks after intervention. Patient was seen in the clinic and reminded not to take any food, water, or smoke $1 \mathrm{~h}$ prior to saliva collection. After mouth-rinsing with $10 \mathrm{mls}$ sterile water at room temperature, unstimulated whole saliva (UWS) was collected by putting 2 pieces of standardized dental roll underneath the patient's tongue. The dental rolls were pre-weighed prior to placing it in the patient's mouth. After $5 \mathrm{~min}$, the dental rolls were removed and weighed; patients were also told to spit any
Fig. 2 Sample A and sample B mouthwashes dispensed to the study population

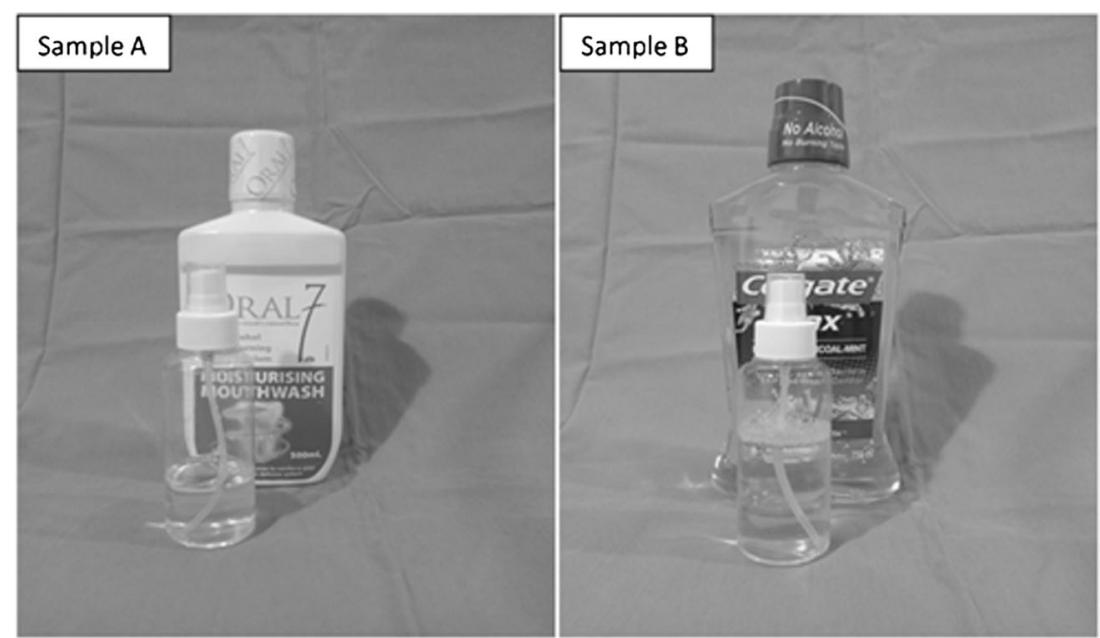


Table 2 Summated xerostomia inventory (SXI) questions

\begin{tabular}{lll}
\hline Item & Question & $\begin{array}{l}\text { Score } \\
\text { (Never (1), } \\
\text { Occasionally } \\
(2), \text { Often (5)) }\end{array}$ \\
\hline 1 & My mouth feels dry when eating a meal & \\
2 & My mouth feels dry & \\
3 & I have difficulty in eating dry foods & \\
5 & I have difficulty swallowing certain foods & \\
& My lips feel dry & \\
\hline
\end{tabular}

amount of saliva in their mouth to a pre-weighed container. Patients were supervised by the researcher and reminded not to swallow any saliva during the collection period. The weight of the UWS was recorded in milligram (mg). The salivary flow rate was expressed as $\mathrm{ml} / \mathrm{min}$, according to the method described by Navazesh and Christensen [20]. Measurements were made immediately after saliva collection. The difference between the UWS measurement taken at baseline and after 4 weeks of intervention was calculated and used as an outcome measure.

Statistical analysis was performed using SPSS statistical analysis software, version 23.0 (SPSS Inc., Chicago, IL). Test for normality was performed using the Shapiro-Wilk test. Since all parameters did not show a normal distribution, nonparametric statistics were applied to group demographic analysis, SXI and UWS difference. Descriptive analysis was carried out and variables were described as median (IQR). Comparison of primary and secondary end points (SXI and UWS difference) was performed using Mann-Whitney $U$ test. Multiple group comparisons were performed using Fisher's exact and Kruskall-Wallis test. $p<0.05$ at $95 \%$ CI was regarded as statistically significant.

\section{Results}

\section{Demographic characteristics}

Ninety-eight patients who fulfilled the inclusion and exclusion criteria were enrolled in this study. Four patients were excluded, making a final study population of 94 patients. Two patients (one from control and interventional arm each) had to be excluded from the study as they could not attend the follow-up visit despite multiple reminders, and the other two (one from control and interventional arm each) requested to be withdrawn from the study 2 weeks after enrolment. One patient from the interventional arm was unable to comply with the instructions given while the other patient from the control arm did not like the taste of the placebo mouthwash. Data from a total of 47 patients in the interventional and control arm, each were analyzed. The median age was 58 years (range, 27-81 years). Among 94 patients, 63 were men (67.0\%) and 31 were women $(33.0 \%)$. The patients were from different ethnicities in which 26 were Chinese $(59.1 \%)$ and 14 were Malay $(31.8 \%)$. Shapiro-Wilk test showed that the data was not normally distributed; hence, non-parametric tests were used for subsequent data analyses.

Table 3 summarizes demographic characteristics, Karnofsky performance score, highest level of education, heterogeneous radiological staging, treatment received, and years after completion of treatment in our study population. Fisher's exact test comparing specific characteristics like gender, ethnicity, age group, Karnofsky performance score, highest level of education, and staging and type of treatment received did not reveal any significant differences between the control and interventional arms $(p>0.05)$. Analysis using Kruskal-Wallis test was performed for years after completion of treatment. No significant differences were seen comparing these potential confounders between the control and interventional arms. The study population was homogeneously distributed across the 2 arms of the study with no significant differences found between specific demographic characteristics (Table 3).

\section{SXI score}

Measurements taken at baseline showed that, majority of patients scored "Often" for the question "I have difficulty in eating dry foods" in both arms. Meanwhile, 98\% of the patient scored "Never" for the question "My lips feel dry." Median of baseline SXI score for the control arm was $19(\mathrm{IQR}=2)$ similar to the interventional arm at $19(\mathrm{IQR}=4)($ Fig. 3). No significant difference in baseline SXI score was found between the 2 arms $(p=0.658)$. This meant the severity of xerostomia between both groups were identical at baseline. At 4 weeks following intervention, the median of total SXI score for the control arm was $16(\mathrm{IQR}=3.5)$, meanwhile for the interventional arm was $14(\mathrm{IQR}=2.5)$. Significant difference was seen in between SXI score at 4 weeks between the 2 arms $(p=0.002)$. SXI difference between 4 weeks and baseline was calculated for all patients (SXI difference $=$ total SXI score at 4 weeks - total SXI score at baseline). The median difference of SXI was $2(\mathrm{IQR}=1)$ for control arm while for interventional arm, the median difference was $4(\mathrm{IQR}=2.5)$ (Fig. 3). All 47 patients in the interventional arm had improved xerostomia symptoms at 4 weeks in contrast to 2 patients in the control arm who had no improvement in xerostomia symptoms. $p$ value obtained using MannWhitney $U$ test showed a significant difference between the 2 arms $(p<0.0001)$. Patients in the interventional arm showed substantial improvement in subjective xerostomia symptoms compared to those in the control arm. 
Table 3 Demographic characteristics of study population

\begin{tabular}{|c|c|c|c|c|}
\hline Demographic characteristic & $\begin{array}{l}\text { Control } \\
n=47 \\
\text { no. of patients }(\%)\end{array}$ & $\begin{array}{l}\text { Interventional } \\
n=47 \\
\text { no. of patients }(\%)\end{array}$ & $\begin{array}{l}\text { Overall } \\
n=94 \\
\text { no. of patients }(\%)\end{array}$ & $p$ value \\
\hline \multicolumn{5}{|l|}{ Gender } \\
\hline $\begin{array}{l}\text { Male } \\
\text { Female }\end{array}$ & $\begin{array}{l}34(72.3 \%) \\
13(27.7 \%)\end{array}$ & $\begin{array}{l}29(61.7 \%) \\
18(38.3 \%)\end{array}$ & $\begin{array}{l}63(67.0 \%) \\
31(33.0 \%)\end{array}$ & $0.739 *$ \\
\hline \multicolumn{5}{|l|}{ Ethnic } \\
\hline $\begin{array}{l}\text { Malay } \\
\text { Chinese }\end{array}$ & $\begin{array}{l}10(21.3 \%) \\
37(78.7 \%)\end{array}$ & $\begin{array}{l}15(31.9 \%) \\
32(68.1 \%)\end{array}$ & $\begin{array}{l}25(26.6 \%) \\
69(73.4 \%)\end{array}$ & $1.000^{*}$ \\
\hline \multicolumn{5}{|l|}{ Age group } \\
\hline $\begin{array}{l}\text { Young and Middle Adult } \\
\text { Elderly }\end{array}$ & $\begin{array}{l}33(70.2 \%) \\
14(29.8 \%)\end{array}$ & $\begin{array}{l}38(80.8 \%) \\
9(19.2 \%)\end{array}$ & $\begin{array}{l}71(75.6 \%) \\
23(24.4 \%)\end{array}$ & $0.337 *$ \\
\hline \multicolumn{5}{|l|}{ Karnofsky performance score } \\
\hline $\begin{array}{l}80 \\
90 \\
100\end{array}$ & $\begin{array}{l}15(31.9 \%) \\
29(61.7 \%) \\
3(6.4 \%)\end{array}$ & $\begin{array}{l}4(8.5 \%) \\
37(78.7 \%) \\
6(12.8 \%)\end{array}$ & $\begin{array}{l}19(20.2 \%) \\
66(70.2 \%) \\
9(9.6 \%)\end{array}$ & $0.931 *$ \\
\hline \multicolumn{5}{|l|}{ Highest education } \\
\hline $\begin{array}{l}\text { Primary } \\
\text { Secondary } \\
\text { Tertiary }\end{array}$ & $\begin{array}{l}9(19.1 \%) \\
26(55.3 \%) \\
12(25.5 \%)\end{array}$ & $\begin{array}{l}9(19.1 \%) \\
28(59.6 \%) \\
10(21.3 \%)\end{array}$ & $\begin{array}{l}17(38.6 \%) \\
4(9.1 \%) \\
2(4.6 \%)\end{array}$ & $0.838^{*}$ \\
\hline \multicolumn{5}{|l|}{ Stage } \\
\hline $\begin{array}{l}\text { I } \\
\text { II } \\
\text { III } \\
\text { IV }\end{array}$ & $\begin{array}{l}8(17.0 \%) \\
22(46.8 \%) \\
15(31.9 \%) \\
2(4.3 \%)\end{array}$ & $\begin{array}{l}4(8.5 \%) \\
25(53.2 \%) \\
14(29.8 \%) \\
4(8.5 \%)\end{array}$ & $\begin{array}{l}11(25.0 \%) \\
1(2.3 \%) \\
2(4.6 \%) \\
2(4.6 \%)\end{array}$ & $0.562 *$ \\
\hline \multicolumn{5}{|l|}{ Treatment received } \\
\hline $\begin{array}{l}\text { Radiotherapy alone } \\
\text { Concurrent chemoradiation }\end{array}$ & $\begin{array}{l}3(6.4 \%) \\
44(93.6 \%)\end{array}$ & $\begin{array}{l}5(10.6 \%) \\
42(89.4 \%)\end{array}$ & $\begin{array}{l}8(8.5 \%) \\
86(91.5 \%)\end{array}$ & $1.000 *$ \\
\hline \multicolumn{5}{|l|}{ Years after completed treatment } \\
\hline $\begin{array}{l}0-5 \\
5-10 \\
>10\end{array}$ & $\begin{array}{l}26(55.3 \%) \\
15(31.9 \%) \\
6(12.8 \%)\end{array}$ & $\begin{array}{l}26(55.3 \%) \\
8(17.0 \%) \\
13(27.7 \%)\end{array}$ & $\begin{array}{l}52(55.3 \%) \\
23(24.5 \%) \\
19(20.2 \%)\end{array}$ & $0.876^{\dagger}$ \\
\hline
\end{tabular}

*Fisher's exact test

${ }^{\dagger}$ Kruskall-Wallis test

\section{Sialometry value}

Median of baseline UWS flow for the control arm was 0.249 $(\mathrm{IQR}=0.389) \mathrm{ml} / \mathrm{min}$ and $0.348(\mathrm{IQR}=0.535) \mathrm{ml} / \mathrm{min}$ for interventional arm (Fig. 4). No significant difference in baseline UWS flow was found between the 2 arms $(p=0.157)$. At 4 weeks following intervention, median UWS flow for the control arm was 0.240 (IQR $=0.389$ ), meanwhile for the interventional arm was 0.453 (IQR $=0.535$ ). Significant difference was seen in between UWS flow at 4 weeks between the 2 arms $(p=0.006)$. UWS difference between 4 weeks and baseline was calculated for all patients (UWS difference $=$ UWS flow at 4 weeks - UWS flow at baseline). The median difference of UWS was $0.002(\mathrm{IQR}=0.005) \mathrm{ml} / \mathrm{min}$ for control arm while for interventional arm the median difference was 0.046 $(\mathrm{IQR}=0.101) \mathrm{ml} / \mathrm{min}$ (Fig. 4). All 47 patients in the interventional arm had improved unstimulated whole salivary flow at
4 weeks in contrast to 11 patients in the control arm who suffered worsening of unstimulated whole salivary flow. $p$ value obtained using Mann-Whitney $U$ test showed a significant difference between the 2 arms $(p<0.0001)$. Patients in the interventional arm showed substantial improvement in unstimulated whole salivary flow compared to those in the control arm.

\section{Harms or unintended effects}

Throughout the study duration, no unintended harms were encountered following the use of either samples A or B among our study population. All patients were compliant to the use of the study samples. There were no reported allergies with the use of the samples throughout the 4 weeks'. However, one patient randomized to the control arm decided to drop out from the study after using sample B for 2 weeks. The reason 


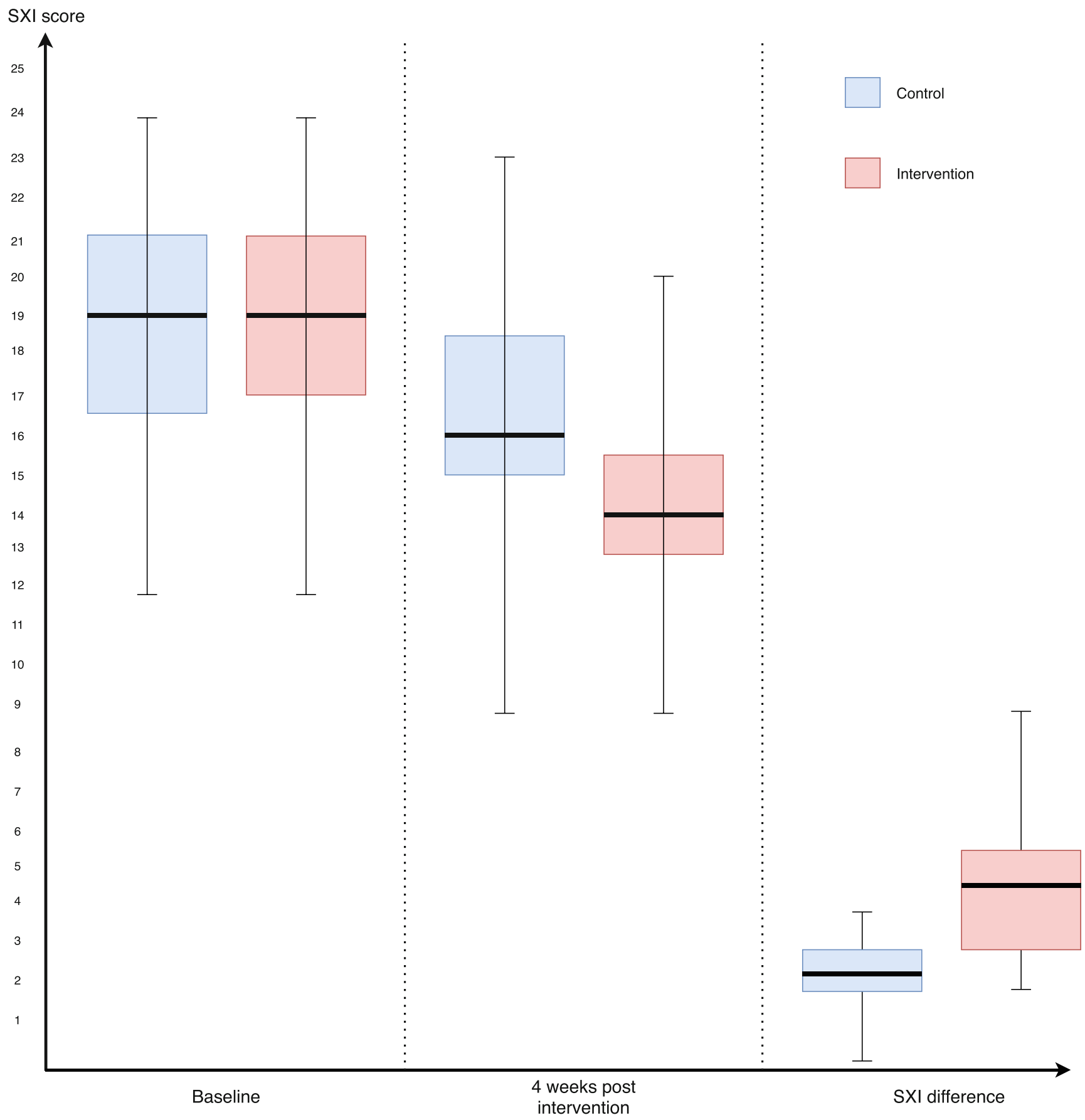

Fig. 3 Box plot showing SXI comparison between treatment arms

for withdrawal was unpleasant taste profile for sample B. This unintended effect was short lived and stopped immediately following discontinuation of treatment. No hospitalization was necessary and no other side effects were documented.

\section{Discussion}

Xerostomia is an important and prevalent complication seen among NPC survivors with a reported prevalence ranging 80
$100 \%$ [6-9]. It is a subjective symptom caused by reduced or absent saliva. Xerostomia leads to impaired chewing, swallowing, altered sense of taste, and speech in NPC survivors. This would eventually affect their nutritional status and ultimately the quality of life. Various treatment strategies for the management of xerostomia have been proposed in the past to reduce patients' symptoms and/or increase salivary flow. Medications such as mucosal lubricants, saliva substitutes, saliva stimulants, and systemic/local sialogogues have been proposed in recent literature [11-13]. 


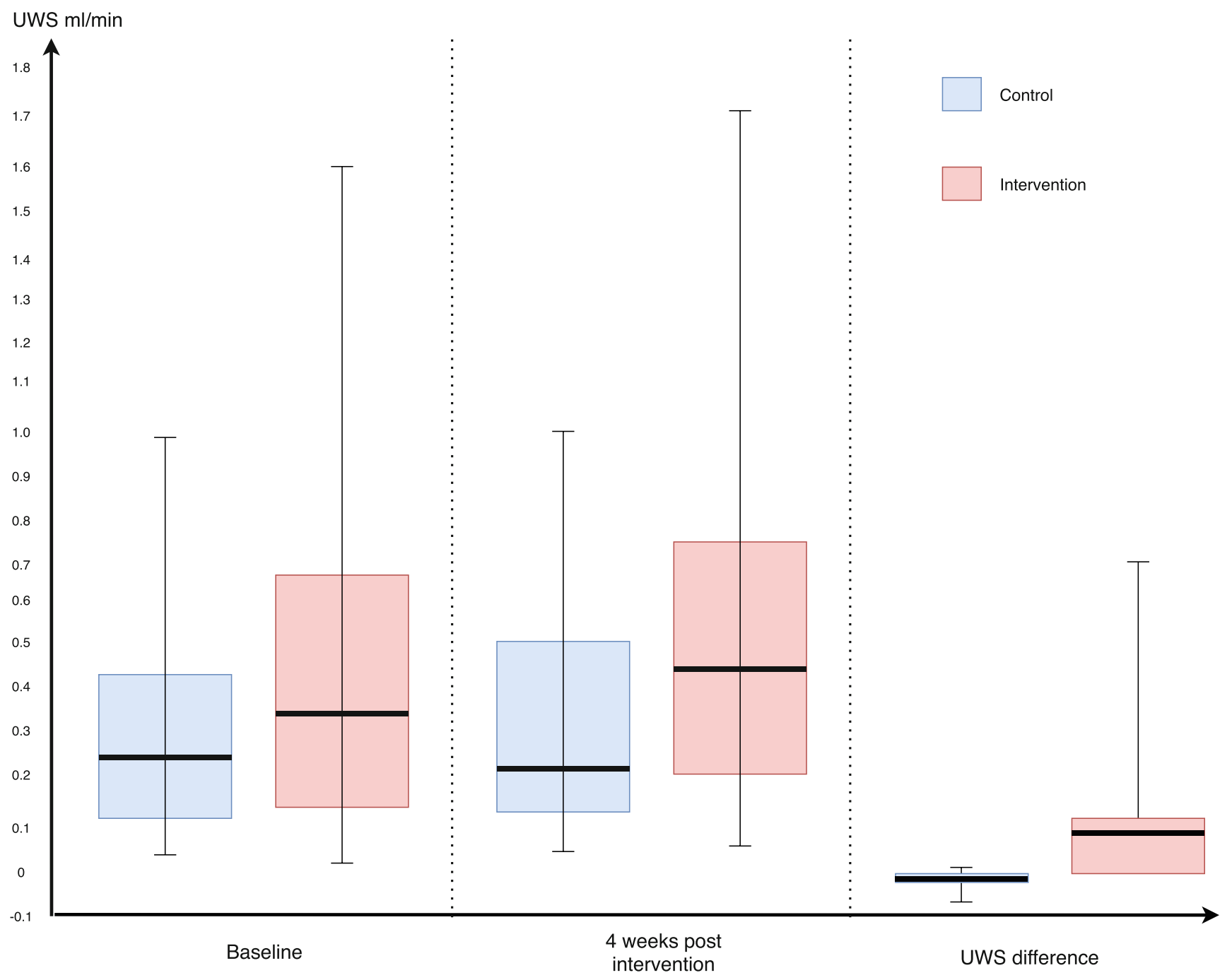

Fig. 4 Box plot showing UWS comparison between treatment arms

Our study compared the efficacy of the saliva substitute Oral7 ${ }^{\circledR}$ mouthwash against placebo in treating xerostomia post radiotherapy in NPC patients. The results support our hypothesis that immunologically active saliva substitute (IASS) can reduce xerostomia symptoms in NPC patients post radiotherapy. Both the subjective and objective outcome measures in the intervention arm showed a statistically significant difference compared to the control arm. SXI scores in patients using Oral7 $®$ showed a significant reduction in all patients with a median of $4(\mathrm{IQR}=2.5)$. Meanwhile, for patients in the control group, there was a reduction of scores in certain patients with a median of $2(\mathrm{IQR}=1)$. Patients in the Oral7 ${ }^{\circledR}$ arm showed an improvement of UWS with a median difference of $0.046(\mathrm{IQR}=0.101) \mathrm{ml} / \mathrm{min}$ compared to those in the control arm where the median UWS difference was 0.002 $(\mathrm{IQR}=0.005) \mathrm{ml} / \mathrm{min}$. This shows Oral7® not only improved patients' xerostomia symptoms but also increased their salivary flow.
The present study was a randomized, placebo-controlled trial studying the efficacy of a saliva substitute mouthwash conducted in a tertiary center. Potential confounders like age group, years after completion of treatment, Karnofsky performance score, tumor stage, and type of treatment received were compared using Fisher's exact test and Kruskal-Wallis test between the two treatment arms. All mentioned confounders were homogenously distributed between the interventional and control arms.

We compared Oral7 ${ }^{\circledR}$ mouthwash used by patients allocated to the interventional arm, and Colgate Plax ${ }^{\circledR}$ used by patients allocated to the placebo arm. Colgate Plax ${ }^{\circledR}$ does not contain natural enzymes, but it possesses biocidal properties with some moisturizing effect, advantageous to our study population. Although physical properties such as color and taste profiles are not exact matches to Oral7 ${ }^{\circledR}$ mouthwash, further blinding was provided by repackaging the two solutions into similar non-pressurized pumps, making it hard for the patients to identify these different mouthwashes. Colgate Plax $®$ is a suitable placebo 
to be used considering that it is not a recognized treatment for xerostomia. The present study is the first to use Colgate Plax® as a placebo. Other published studies have used sodium fluoride solution and salt-soda mouthwash as placebo [21, 22].

A pilot study of 30 patients by Jellema et al. studied the efficacy of xialine, a type of saliva substitute in a placebocontrolled randomized cross-over study using EORTC QLQC30 and the QLQ-H\&N35 questionnaires. In this study, a small but not statistically significant improvement was demonstrated in the global quality of life (QoL) scores after 1 week of treatment with either xialine or placebo [23]. Although the cross-over trial design was better compared to the present study, the duration of treatment was perceived to be too short, and desired outcomes could not be observed. The present study recruited more patients and increased the treatment duration (4 weeks) to rigorously assess the effects of the saliva substitute Oral7® mouthwash. Future cross-over trials utilizing similar or longer treatment durations would consolidate the findings of our study, but could suffer from higher drop out.

The summated xerostomia inventory was used in this present study as a primary outcome measure. This inventory was adapted from the SXI-Dutch version which has been shown to be a valid tool for measuring xerostomia symptoms in a clinical research [19]. Following validation of the summated English version, it has been used widely in many clinical and epidemiological studies on xerostomia $[24,25]$. In the present study, this inventory was administered using a structured interview by a dedicated researcher, which exposes the study to the risk of evaluation apprehension. To utilize a patient-administered questionnaire, the multi ethnic study population called for a validated inventory in Malay and Mandarin languages which was unavailable at the time of study design [26, 27]. Validation of the SXI-Dutch version in Malay and Mandarin languages is a potential avenue for further research as it will facilitate the delivery of this inventory as an outcome measure in future trials. In contrast to QLQH\&N35 questionnaires, the summated xerostomia inventory has more focused questions addressing xerostomia symptoms, making it less time consuming for the patients. The absence of global QoL scores for further analysis did not affect the study results as it is well documented that xerostomia adversely affects QoL outcomes in NPC survivors [10].

Various methods for objective measurement of salivary flow, an important outcome measure in xerostomia research have been reported and tested for validity and reproducibility [20, 28-30]. The present study measured unstimulated whole saliva (UWS) using a method described by Navazesh and Christensen. Measurement of salivary flow from individual salivary glands, although more accurate is time consuming, laborious, and require custom-made collection devices [31]. Additionally, cannulating the salivary ducts can potentially introduce ascending infection to our already vulnerable study population. A later systematic review by Löfgren et al. evaluating methods to measure salivary flow using Quality Assessment of Diagnostic Accuracy Studies
(QUADAS) was unable to conclude a gold standard method of evaluating salivary flow [32]. Although the original study by Navazesh and Christensen reported only acceptable reliability (test-retest correlation coefficient $=0.68$ ) using the UWS collection method, the study population consisted of only 17 healthy volunteers. During conception of this current study, UWS was chosen as an outcome measure because it is non-invasive, less time consuming and widely used in other published literature [22, 33-36].

Normal salivary flow is an important prerequisite for homeostasis of the oral environment. With the introduction of radiation to treat malignant pathologies of the upper aerodigestive tract, the patient will suffer loss of 50-70\% of saliva function if 10-15 Gy of radiation is applied or even worse it is undetectable when higher doses of radiation ( $40-42 \mathrm{~Gy}$ ) is given to the patient [37]. There have been numerous published studies on the efficacy of saliva substitute in treating xerostomia [14, 38-44]. Studies that looked into the efficacy of Oral7 $₫$ mouthwash in treating xerostomia in post radiotherapy patients is, however scarce [21]. Bachok et al. in their preliminary randomized trial of 30 patients undergoing radiotherapy for head and neck cancer comparing Oral7® mouthwash and salt-soda mouthwash reported significant improvement in QoL scores at 3 months post-treatment. Although the study included sialometry measurements as secondary end points, this outcome measure was not reported. The findings of our study showed that at 4 weeks post intervention, patients treated with Oral7® mouthwash had a median UWS of $0.453(\mathrm{IQR}=0.535) \mathrm{ml} / \mathrm{min}$, which was significantly higher compared to those treated with placebo. As many as $23(49 \%)$ patients in the interventional arm achieved a normal UWS more than $0.5 \mathrm{ml} / \mathrm{min}$ at 4 weeks post intervention. With these findings, we believe that Oral7® mouthwash is beneficial to maintain the unstimulated salivary flow to a near-normal state.

Findings of this study are consistent with most previous studies on saliva substitute used in various study populations with xerostomia [39-43]. The saliva substitute tested comprised agents such as immunologically active natural enzymes (current study), carboxylmethy cellulose, mucin, and glycerin $[14,40-43]$. To date, excluding the earlier trial by Bachok et al., there are two other studies investigating the efficacy of immunologically active natural enzymes. BioXtra gel containing lysozyme was studied in a non-randomized trial among 58 patients with head and neck cancer where improved xerostomia symptom score after 2 weeks use was reported [45]. Another randomized study in 134 diabetic patients treated with 6 months of immunologically active natural enzymes showed significant improvement in dental plaque index and yeast culture, which may shed light to additional benefit of long term treatment with this type of saliva substitute [44]. Findings of these studies corroborated that immunologically active natural enzymes is a safe and efficacious salivary substitute to use in various study population with xerostomia [14, $21,44,45]$. At the time of writing, the present study was the 
first to document improved UWS flow with 4 weeks' use of immunologically active natural enzymes.

Our results support the clinical use of Oral $7 \AA$ mouthwash in alleviating xerostomia symptoms among NPC survivors. Hence, we would like to recommend continued use of the saliva substitute to obtain desired clinical outcomes in these patients. The findings of this study can be extrapolated for clinical application in other diseases presenting with xerostomia such as Sjögren syndrome or drug induced xerostomia. However, we did not evaluate whether sustained effects can be seen following discontinuation of use; hence, this cannot be considered a permanent treatment. Albeit this treatment avenue does not require any surgical intervention to alter the glands such as glandopexy or tissue regenerative methods, the patient will have to use the product regularly throughout their lifetime.

\section{Conclusion}

Immunologically active saliva substitute (IASS) significantly reduces subjective xerostomia scores measured using SXI and improves objective measurement of salivary flow using UWS among nasopharyngeal cancer survivors with xerostomia. Immunologically active saliva substitute (IASS) is significantly more effective in improving subjective and objective xerostomia measurements compared to non-immunologically active mouthwash. Additionally, this treatment is very safe, with superior side effect profiles.

Acknowledgments The authors would like to thank Mrs Zunaidah Hashim, registered nurse, UKM Medical Centre, who was involved in dispensing the samples to the patients.

Author contributions All authors contributed to the study conception and design. Material preparation, data collection and analysis were performed by Dr Dayaabaran Marimuthu and Dr Mawaddah Azman. The first draft of the manuscript was written by Dr Dayaabaran Marimuthu and all authors commented on previous versions of the manuscript. All authors read and approved the final manuscript.

Funding The work was supported by the Department of Otorhinolaryngology-Head and Neck Surgery, National University of Malaysia with funding from the Fundamental Grant (FF-2018-133). This study also recieved support from the Centre of Studies for Oral and Maxillofacial Surgery, Faculty of Dentistry, UiTM Sungai Buloh Campus, Malaysia.

\section{Compliance with ethical standards}

Conflict of interest The authors declare that they have no conflict of interest.

Ethical approval All procedures performed in this study involving human participants were in accordance with the ethical standards of the institutional and/or national research committee and with the 1964 Helsinki declaration and its later amendments or comparable ethical standards. The conduct of this study was approved by the institutional Ethical and Review Board (FF-2018-133) prior to commencement of the study.

Informed consent Informed consent was obtained from all individual participants included in the study.

Disclaimer The study did not receive any financial contribution from the distributor or manufacturer of Oral7 $®$ mouthwash and none of the authors are affiliated to the company MBD Marketing Malaysia. The protocol is publicly available at ClinicalTrials.gov and can be traced using the identifier: NCT04491435.

Open Access This article is licensed under a Creative Commons Attribution 4.0 International License, which permits use, sharing, adaptation, distribution and reproduction in any medium or format, as long as you give appropriate credit to the original author(s) and the source, provide a link to the Creative Commons licence, and indicate if changes were made. The images or other third party material in this article are included in the article's Creative Commons licence, unless indicated otherwise in a credit line to the material. If material is not included in the article's Creative Commons licence and your intended use is not permitted by statutory regulation or exceeds the permitted use, you will need to obtain permission directly from the copyright holder. To view a copy of this licence, visit http://creativecommons.org/licenses/by/4.0/.

\section{References}

1. Pua KC, Khoo AS, Yap YY, Subramaniam SK, Ong CA, Gopala Krishnan G et al (2008) Nasopharyngeal Carcinoma Database. Med J Malaysia 63 Suppl C:59-62

2. Prasad U, Pua KC (2000) Nasopharyngeal carcinoma: a delay in diagnosis. Med J Malaysia 55:230-235

3. Suzina SA, Hamzah M (2003) Clinical presentation of patients with nasopharyngeal carcinoma. Med J Malaysia 58:539-545

4. Pinna R, Campus G, Cumbo E, Mura I, Milia E (2015) Xerostomia induced by radiotherapy: an overview of the physiopathology, clinical evidence, and management of the oral damage. Ther Clin Risk Manag 11:171-188

5. Vissink A, Mitchell JB, Baum BJ, Limesand KH, Jensen SB, Fox PC, Elting LS, Langendijk JA, Coppes RP, Reyland ME (2010) Clinical management of salivary gland hypofunction and xerostomia in head-and-neck cancer patients: successes and barriers. Int J Radiat Oncol Biol Phys 78:983-991

6. Wang WC, Chen YK, Lin LM (2008) Oral care experiences with 181 nasopharyngeal carcinoma patients receiving radiotherapy in a Taiwanese hospital. Auris Nasus Larynx 35:230-234

7. Porter SR, Scully C, Hegarty AM (2004) An update of the etiology and management of xerostomia. Oral Surg Oral Med Oral Pathol Oral Radiol Endod 97:28-46

8. Schwarz E, Chiu GK, Leung WK (1999) Oral health status of southern Chinese following head and neck irradiation therapy for nasopharyngeal carcinoma. J Dent 27:21-28

9. Epstein JB, Emerton S, Lunn R, Le N, Wong FL (1999) Pretreatment assessment and dental management of patients with nasopharyngeal carcinoma. Oral Oncol 35:33-39

10. Leong KY, Azman M, Kong MH (2020) Quality of life outcome among nasopharyngeal carcinoma survivors in Kuala Lumpur. Annals Of Nasopharynx Cancer. https://doi.org/10.21037/anpc20-14

11. Villa A, Connell CL, Abati S (2015) Diagnosis and management of xerostomia and hyposalivation. Ther Clin Risk Manag 11:45-51 
12. Lee CC, Ho CY (2012) Post-treatment late complications of nasopharyngeal carcinoma. Eur Arch Otorhinolaryngol 269:2401-2409

13. Jensen SB, Pedersen AM, Vissink A, Andersen E, Brown CG, Davies AN et al (2010) A systematic review of salivary gland hypofunction and xerostomia induced by cancer therapies: management strategies and economic impact. Support Care Cancer 18: 1061-1079

14. Assery MKA (2019) Efficacy of artificial salivary substitutes in treatment of xerostomia: a systematic review. J Pharm Bioallied Sci 11:S1-S12

15. Hahnel S, Behr M, Handel G, Burgers R (2009) Saliva substitutes for the treatment of radiation-induced xerostomia-a review. Support Care Cancer 17:1331-1343

16. Chan AW, Altman DG (2005) Epidemiology and reporting of randomised trials published in PubMed journals. Lancet. 365: $1159-1162$

17. Erikson EH (1956) The problem of ego identity. J Am Psychoanal Assoc 4:56-121

18. Doescher J, Veit JA, Hoffmann TK (2017) The 8th edition of the AJCC Cancer Staging Manual : Updates in otorhinolaryngology, head and neck surgery. HNO 65:956-961

19. Thomson WM, van der Putten GJ, de Baat C, Ikebe K, Matsuda K, Enoki K, Hopcraft MS, Ling GY (2011) Shortening the xerostomia inventory. Oral Surg Oral Med Oral Pathol Oral Radiol Endod 112: 322-327

20. Navazesh M, Christensen CM (1982) A comparison of whole mouth resting and stimulated salivary measurement procedures. J Dent Res 61:1158-1162

21. Bachok N, Biswal BM, Razak NHA, Zainoon W, Mokhtar K, Rahman RA et al (2018) Preliminary comparative study of Oral7(R) versus salt-soda mouthwash on oral health related problems and quality of life among head and neck cancer patients undergoing radiotherapy. Malays J Med Sci 25:79-87

22. Sweeney MP, Bagg J, Baxter WP, Aitchison TC (1997) Clinical trial of a mucin-containing oral spray for treatment of xerostomia in hospice patients. Palliat Med 11:225-232

23. Jellema AP, Langendijk H, Bergenhenegouwen L, van der Reijden W, Leemans R, Smeele L, Slotman BJ (2001) The efficacy of xialine in patients with xerostomia resulting from radiotherapy for head and neck cancer: a pilot-study. Radiother Oncol 59:157-160

24. Tashbayev B, Garen T, Palm O, Chen X, Herlofson BB, Young A, Hove LH, Rykke M, Singh PB, Aqrawi LA, Utheim ØA, Utheim TP, Jensen JL (2020) Patients with non-Sjogren's sicca report poorer general and oral health-related quality of life than patients with Sjogren's syndrome: a cross-sectional study. Sci Rep 10:2063

25. Chapuis J, Siu-Paredes F, Pavageau C, Amador G, Rude N, Denis F (2020) Anticholinergic drugs and oral health-related quality of life in patients with schizophrenia: a pilot study. Transl Neurosci 11: $10-16$

26. Amaral J, Marques D, Thomson WM, Vinagre ARR, da Mata A (2018) Validity and reliability of a Portuguese version of the Summated Xerostomia Inventory-5. Gerodontology. 35:33-37

27. Hohenberger R, Baumann I, Plinkert PK, Brinster R, Krisam J, Affolter A, Hein M, Flechsig P, Rathke H, Oladokun D, Lippert BM, Bulut OC (2019) Validating the Xerostomia Inventory in a radiation-induced xerostomia population in German language. Oral Dis 25:1744-1750

28. Chambers MS, Posner M, Jones CU, Biel MA, Hodge KM, Vitti R, Armstrong I, Yen C, Weber RS (2007) Cevimeline for the treatment of postirradiation xerostomia in patients with head and neck cancer. Int J Radiat Oncol Biol Phys 68:1102-1109

29. Hargitai IA, Sherman RG, Strother JM (2005) The effects of electrostimulation on parotid saliva flow: a pilot study. Oral Surg Oral Med Oral Pathol Oral Radiol Endod 99:316-320
30. Jones JM, Watkins CA, Hand JS, Warren JJ, Cowen HJ (2000) Comparison of three salivary flow rate assessment methods in an elderly population. Community Dent Oral Epidemiol 28:177-184

31. Navazesh M, Kumar SK (2008) University of Southern California School of D. Measuring salivary flow: challenges and opportunities. J Am Dent Assoc 139 Suppl:35S-40S

32. Lofgren CD, Wickstrom C, Sonesson M, Lagunas PT, Christersson C (2012) A systematic review of methods to diagnose oral dryness and salivary gland function. BMC Oral Health 12:29

33. LeVeque FG, Montgomery M, Potter D, Zimmer MB, Rieke JW, Steiger BW et al (1993) A multicenter, randomized, double-blind, placebo-controlled, dose-titration study of oral pilocarpine for treatment of radiation-induced xerostomia in head and neck cancer patients. J Clin Oncol 11:1124-1131

34. McMillan AS, Tsang CS, Wong MC, Kam AY (2006) Efficacy of a novel lubricating system in the management of radiotherapy-related xerostomia. Oral Oncol 42:842-848

35. Navarro Morante A, Wolff A, Bautista Mendoza GR, Lopez-Jornet P (2017) Natural products for the management of xerostomia: a randomized, double-blinded, placebo-controlled clinical trial. J Oral Pathol Med. 46:154-160

36. Gomez-Moreno G, Cabrera-Ayala M, Aguilar-Salvatierra A, Guardia J, Ramirez-Fernandez MP, Gonzalez-Jaranay M et al (2014) Evaluation of the efficacy of a topical sialogogue spray containing malic acid $1 \%$ in elderly people with xerostomia: a double-blind, randomized clinical trial. Gerodontology. 31:274 280

37. Eisbruch A, Ten Haken RK, Kim HM, Marsh LH, Ship JA (1999) Dose, volume, and function relationships in parotid salivary glands following conformal and intensity-modulated irradiation of head and neck cancer. Int J Radiat Oncol Biol Phys 45:577-587

38. See L, Mohammadi M, Han PP, Mulligan R, Enciso R (2019) Efficacy of saliva substitutes and stimulants in the treatment of dry mouth. Spec Care Dentist 39:287-297

39. Shiboski CH, Hodgson TA, Ship JA, Schiodt M (2007) Management of salivary hypofunction during and after radiotherapy. Oral Surg Oral Med Oral Pathol Oral Radiol Endod 103 Suppl: S66 e1-S66 19

40. Momm F, Volegova-Neher NJ, Schulte-Monting J, Guttenberger R (2005) Different saliva substitutes for treatment of xerostomia following radiotherapy. A prospective crossover study. Strahlenther Onkol 181:231-236

41. Davies AN (2000) A comparison of artificial saliva and chewing gum in the management of xerostomia in patients with advanced cancer. Palliat Med 14:197-203

42. Aagaard A, Godiksen S, Teglers PT, Schiodt M, Glenert U (1992) Comparison between new saliva stimulants in patients with dry mouth: a placebo-controlled double-blind crossover study. J Oral Pathol Med 21:376-380

43. Gravenmade EJ, Vissink A (1993) Mucin-containing lozenges in the treatment of intraoral problems associated with Sjogren's syndrome. A double-blind crossover study in 42 patients. Oral Surg Oral Med Oral Pathol 75:466-471

44. Montaldo L, Montaldo P, Papa A, Caramico N, Toro G (2010) Effects of saliva substitutes on oral status in patients with Type 2 diabetes. Diabet Med 27:1280-1283

45. Gookizadeh A, Emami H, Najafizadeh N, Roayaei M (2012) Clinical evaluation of BIOXTRA in relieving signs and symptoms of dry mouth after head and neck radiotherapy of cancer patients at Seyed-al-Shohada Hospital, Isfahan, Iran. Adv Biomed Res 1:72

Publisher's note Springer Nature remains neutral with regard to jurisdictional claims in published maps and institutional affiliations. 